\title{
ATRIBUTOS FÍSICOS AVALIADOS EM UM LATOSSOLO AMARELO EM DUAS ÉPOCAS DO ANO NO MUNICÍPIO DE CAPITÃO POÇO, PA
}

\author{
Danielly Cristina da Silva Marques ${ }^{1}$; Deymeson Mateus Soares da Silva ${ }^{1}$; Shirlene \\ Souza Oliveira; Jairo dos Santos Reis ${ }^{1}$; Jorge Luís Padilha Costa ${ }^{1}$; Eduardo Cézar \\ Medeiros Saldanha². \\ ${ }^{1}$ Estudante do Curso de Agronomia da Universidade Federal Rural da Amazônia, Campus Capitão Poço. \\ Capitão Poço, Pará, Brasil. marquesdanielly@ hotmail.com \\ ${ }^{2}$ Engenheiro Agrônomo e Professor Assistente da Universidade Federal Rural da Amazônia, Campus Capitão \\ Poço - PA, (Rod. Pa 124, KM 0-Bairro: Vila Nova, Cep 68650-000); e-mail: eduardo.saldanha@ufra.edu.br.
}

RESUMO: A adoção de técnicas racionais de manejo conservacionista do solo e da água é de fundamental importância para a sustentabilidade. Os atributos físicos do solo possuem importância edáfica, uma vez que implicam diretamente na produção agrícola. O objetivo desse trabalho foi avaliar o comportamento de alguns atributos físicos do solo: a lâmina d’água e a densidade do solo, em dois períodos do ano, em um Latossolo Amarelo no município de Capitão Poço, PA. As coletas foram realizadas aleatoriamente nas profundidades $(0-20,20-40,40-60$ e 60-80) em dois períodos consecutivos do ano, seco e chuvoso, respectivamente. $\mathrm{O}$ delineamento experimental utilizado foi o inteiramente casualizado (4 profundidades x 2 períodos de coleta). Os parâmetros físicos analisados neste trabalho apresentaram distinções entre os períodos analisados e quanto à profundidade amostrada.

PALAVRAS-CHAVE: densidade, umidade, perfil do solo.

\section{PHYSICAL ATTRIBUTES EVALUATED IN OXISOL TWO TIMES OF THE YEAR IN THE MUNICIPALITY OF CAPITÃO POÇO, PA}

\begin{abstract}
The adoption of rational conservation management of soil and water is fundamental to sustainability. The soil physical properties have important edaphic, since they involve directly in agricultural production. The aim of this study was to evaluate the behavior of some soil physical properties: the water depths and soil bulk density in two periods of the year in some soil in the municipality of Captain Wells, PA. Samples were collected at random depths (0-20, 20-40, 40-60 and 60-80) in two consecutive periods of the year, dry and rainy seasons, respectively. The experimental design was completely randomized (4 x 2 depths collection periods). The physical parameters analyzed in this study showed distinctions between periods and to the depth sampled.
\end{abstract}

KEY WORDS: density, moisture, profile soil. 


\section{INTRODUÇÃO}

A adoção de técnicas racionais de manejo conservacionista do solo e da água é de fundamental importância para a sustentabilidade, de tal forma que se possa, economicamente, manter ao longo do tempo esses recursos com quantidade e qualidade suficientes para a manutenção de níveis satisfatórios de produtividade (SIQUEIRA, 2006).

O uso agrícola das terras altera, normalmente, as propriedades do solo, dependendo das condições edáficas e climáticas. Dessa forma, diferentes sistemas de manejo resultam em mudanças na composição e arranjo dos constituintes do solo, que podem em alguns casos, prejudicar a conservação desse recurso natural e reduzir a produtividade das culturas (SILVA et al., 2006).

Para Carvalho et al. (2003), o solo apresenta variabilidade espacial e temporal de seus atributos, resultante da interação de processos que comandam os fatores responsáveis pela sua formação. Vieira et al. (1983) consideraram que dentro de um certo domínio, as diferenças entre valores de uma propriedade do solo podem ser expressas como uma função da distância de separação entre pontos medidos.

O conhecimento espacial dos atributos do solo em determinada área é importante para a avaliação dos efeitos da agricultura sobre a qualidade ambiental, assim como é importante para a definição da intensidade de amostragem do solo para sua caracterização, possibilitando, desta maneira, reduzir o erro padrão da média, maximizando a eficiência da amostragem e reduzir os custos e mão-deobra do trabalho (CORÁ et al., 2004). Os atributos físicos do solo possuem uma importância para a caracterização do solo, uma vez que implicam diretamente na produção das culturas.

As variações espaciais dos mais diversos atributos físicos tais como a densidade e a porosidade condicionam a distribuição da água no solo e consequentemente o rendimento das culturas. A medida da umidade é essencial para a pesquisa e entendimento do comportamento de distribuição e armazenamento de água no solo. O clima interfere diretamente no armazenamento de água através dos mais variados fatores como: intensidade $\mathrm{da}$ radiação solar, temperatura do ar, temperatura do solo, vento e umidade do ar. Compreender como estes fatores interferem no armazenamento de água no solo e sua interligação com o próprio solo é um desafio real para a manutenção dos processos de produção agrícola (SIQUEIRA, 2006).

O objetivo desse trabalho foi avaliar alguns atributos físicos do solo em dois 
períodos do ano, em um Latossolo Amarelo no município de Capitão Poço, PA.

\section{MATERIAL E MÉTODOS}

A área de amostragem faz parte da área experimental da Universidade Federal Rural da Amazônia, Campus de Capitão Poço (UFRA), (01 $\left.{ }^{\circ} 44^{\prime} 04^{\prime \prime} \mathrm{S}, 47^{\circ} 03^{\prime} 28^{\prime \prime} \mathrm{OE}\right)$, na mesorregião Nordeste do Estado do Pará.

Com relação ao clima do município, segundo Pacheco e Bastos (2002), enquadrase no tipo climático Ami, da classificação de Köppen, caracterizado chuvoso ou sem inverno estacional, apresentando, porém, pequena estação seca.

A temperatura média situa-se em torno de $26,2{ }^{\circ} \mathrm{C}$, enquanto a umidade relativa fica em torno de $83 \%$ e o solo é classificado como um Latossolo Amarelo distrófico (EMBRAPA, 2006).

A caracterização química e física do solo foi realizada antes da instalação do experimento, coletando-se amostras na profundidade de $0-20 \mathrm{~cm}$. Onde a mesma apresentou os seguintes resultados: $\mathrm{pH}$ $\left(\mathrm{H}_{2} \mathrm{O}\right)=5,1 ; \mathrm{MO}=18$ g.kg ${ }^{-1} ; \mathrm{P}$ (Melich 1) $=1,3 \mathrm{mg} \mathrm{dm}{ }^{-3} ; \mathrm{Ca}^{2+}=1,1 \mathrm{cmol}_{\mathrm{c}} \mathrm{dm}^{-3}$; $\mathrm{Ca}+\mathrm{Mg}^{2+}=1,4 \mathrm{cmol}_{\mathrm{c}} \mathrm{dm}^{-3} ; \mathrm{K}=0,05 \mathrm{cmol}_{\mathrm{c}}$ $\mathrm{dm}^{-3} ; \mathrm{H}+\mathrm{Al}=3,2 \mathrm{cmol}_{\mathrm{c}} \mathrm{dm}^{-3} ; \mathrm{Al}^{3+}=0,3$ $\mathrm{Cmol}_{\mathrm{c}} \mathrm{dm}^{-3} ; \mathrm{CTC}=4,61 \mathrm{cmol}_{\mathrm{c}} \mathrm{dm}^{-3} ; \mathrm{V}=$ $30,6 \% ; \mathrm{m}=25,8 \%$. A análise granulométrica mostrou os seguintes resultados: areia $=733 \mathrm{~g} \mathrm{~kg}^{-1} ;$ argila $=177 \mathrm{~g}$ $\mathrm{kg}^{-1}$ e silte $=89 \mathrm{~g} \mathrm{~kg}^{-1}$, caracterizando uma textura franco-arenosa.

Os atributos físicos avaliados foram: densidade do solo e lâmina de água existente no solo, as coletas foram realizadas aleatoriamente em área de capoeira, nas profundidades (0-20, 20-40, 40-60 e 60-80 cm) em dois períodos consecutivos do ano, seco e chuvoso, respectivamente.

O delineamento experimental utilizado foi o inteiramente casualizado (com quatro profundidades de coleta e em dois períodos).

A densidade do solo foi obtida pela relação entre a massa do solo seco a 105$110^{\circ} \mathrm{C}$ e o volume da amostra considerado igual ao volume do cilindro amostrador (TORMENA et al., 1998) com diâmetro médio de $6,0 \mathrm{~cm}$ e altura $3,7 \mathrm{~cm}$.

Para a lâmina de água, a metodologia consistiu pelo método da umidade gravimétrica (EMBRAPA, 1997).

Os resultados obtidos foram submetidos à estatística descritiva.

\section{RESULTADOS E DISCUSSÃO}

Observou-se que a lâmina de água no solo (Figura 1), no período chuvoso, variou conforme a profundidade de coleta. Sendo em torno de $500 \mathrm{~m}^{3} /$ ha nas profundidades de 0 a $20 \mathrm{~cm}$ e na profundidade de $40-60 \mathrm{~cm}$. 
Enquanto que no período seco, a maior lâmina de água observada, foi na camada superficial do solo, ou seja, de 0-20 cm de profundidade.

O efeito da compactação sobre características físicas do solo, como porosidade e densidade é maior na superfície do solo e pode levar à redução na capacidade de retenção e no conteúdo de água disponível (TORMENA, 1998). A variabilidade espacial e temporal dos atributos dos solos ocorre em diferentes níveis, estando relacionada a fatores tais como: clima, relevo, ação de organismos, tempo, variação do material de origem nos processos genéticos de formação do solo e/ou efeitos de técnicas de manejo (CORREA et al., 2009).

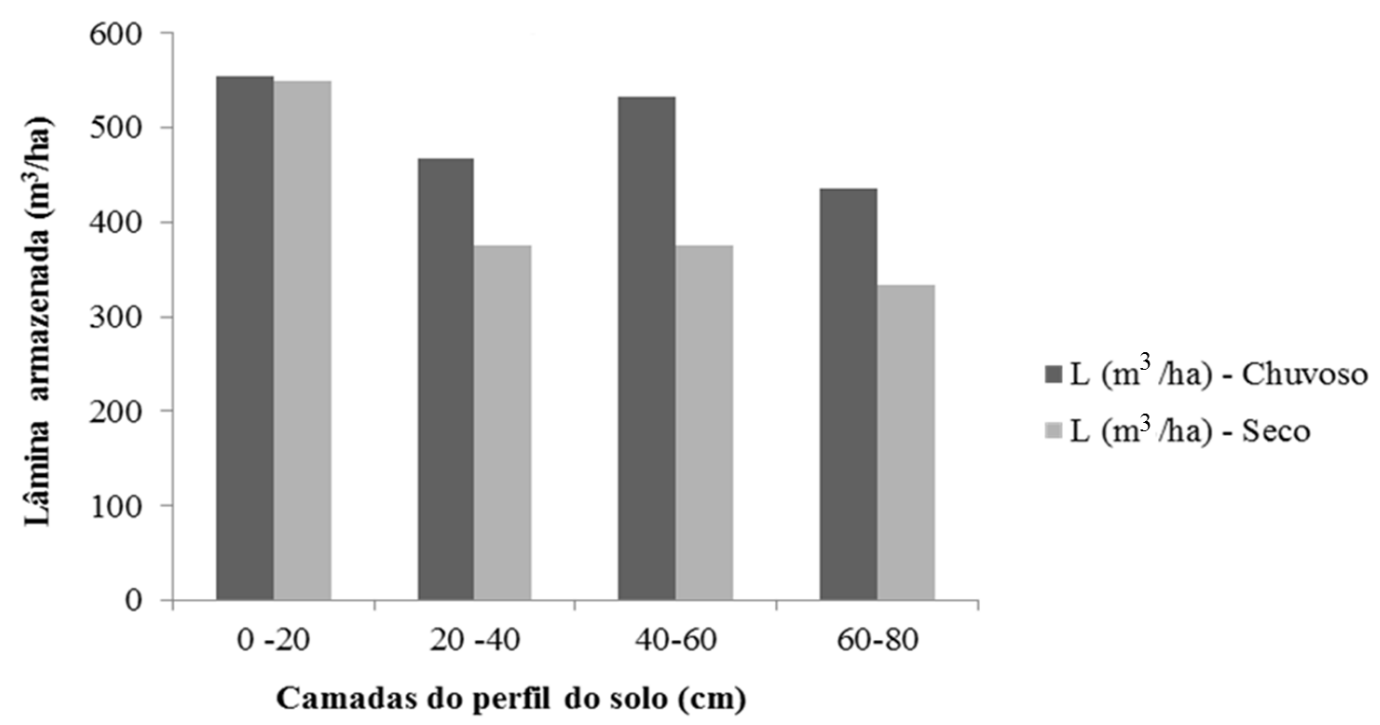

Figura 1. Lâmina de água disponível em função da profundidade, em dois períodos do ano, em Latossolo Amarelo, Capitão Poço, PA.

Quanto à densidade do solo observada (Figura 2), verificou-se que houve comportamento diferente em função dos períodos. No período chuvoso, observa-se que nas camadas de 20 a $40 \mathrm{~cm}$ e 40 a 60 $\mathrm{cm}$, a densidade do solo foi maior. Enquanto que para o período seco, o atributo observado, apresentou um decréscimo desde a camada de 0 a $20 \mathrm{~cm}$, diminuindo gradativamente até o ultimo perfil analisado. Silva et al. (2006), avaliando as modificações das propriedades físicas relacionadas com a estrutura e agregação e teor de $\mathrm{CO}$ de um Argissolo, após 17 anos 
sob sistemas de manejo, em comparação a uma área de campo nativo, observaram que a densidade do solo, variou de 1,35 a 1,67 $\mathrm{kg} \mathrm{dm}^{-3}$, com efeito do sistema de manejo só na profundidade de $2,5-7,5 \mathrm{~cm}$. Parâmetros físicos do solo como a densidade e a porosidade podem indicar problemas significativos de infiltração, permeabilidade, aeração e drenagem (SIQUEIRA, 2006). Já Vieira et al., (2008) não encontraram diferenças da densidade e na porosidade do solo, ao aplicar durante 9 anos de $5 \mathrm{~m}^{3} / \mathrm{ha} /$ ano de cama de aviário, $40 \mathrm{~m}^{3} / \mathrm{ha} /$ ano de densidade laminar $60 \mathrm{~m}^{3} / \mathrm{ha} / \mathrm{ano}$ de dejeto líquido de bovinos num solo muito argiloso.

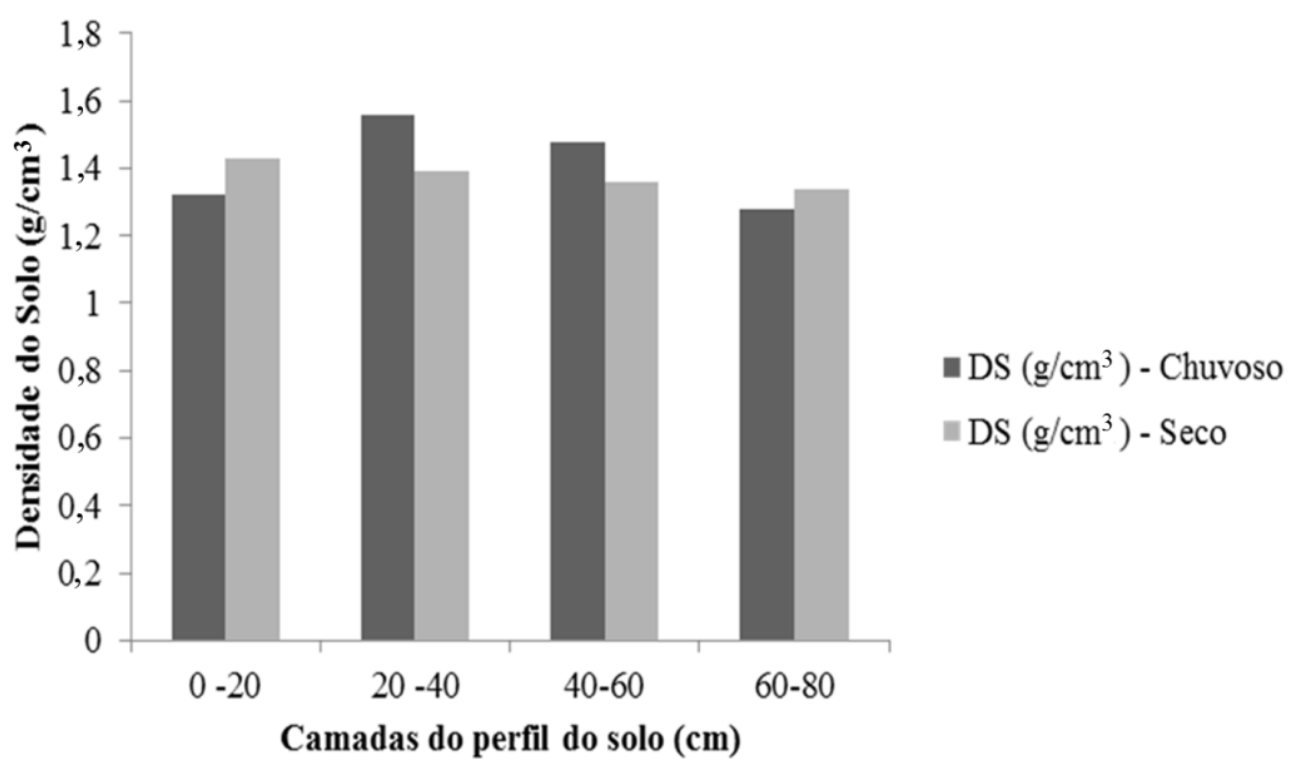

Figura 2. Densidade do solo em função da profundidade, em dois períodos do ano, em Latossolo Amarelo, Capitão Poço, PA.

\section{CONCLUSÃO}

Os parâmetros físicos analisados neste trabalho apresentaram modificações em função tanto do período analisado, quanto na profundidade.
As lâminas de água foram maiores no período chuvoso. Enquanto que no período seco, foi maior para camada de 0-20 (cm).

A densidade do solo foi maior no período seco na camada arável $(0-20 \mathrm{~cm})$. 


\section{REFERÊNCIAS}

CLAESSEN, E. C. (Coord.). Manual de métodos de análise de solo. 2ed. Rio de Janeiro: Embrapa CNPS, 1997. 212p. (Documento,1). Disponível em: $<$ http://www.agencia.cnptia.embrapa.br/Rep ositorio/Manual+de+Metodos_000fzvhotqk 02wx5ok0q43a0ram31wtr.pdf>. Acesso em: 30 jun. 2013.

CORREAA， A.N.; TAVARES， M.H.F.; URIBE-OPAZO, M.A. Variabilidade espacial de atributos físicos do solo e seus efeitos sobre a produtividade do trigo. Semina: ciências agrárias, Londrina, v. 30, n.1 p. 81-94, 2009. Disponível em: <http://www.uel.br/revistas/uel/index.php/se magrarias/article/viewArticle/2634>. Acesso em: 29 jun. 2013.

SIQUEIRA, G. M.; VIEIRA, S. R.; CEDDIA, M. Variabilidade de atributos físicos do solo determinados por métodos diversos. Bragantia, Campinas, v. 67, n. 1, 2008. Disponível em: $<$ http://www.scielo.br/scielo.php?script=sci _arttext\&pid=S0006$87052008000100025 \& \operatorname{lng}=e n \& n r m=i s o>$. Acesso em: 01 jul. 2013.

TORMENA, C.A.; ROLOF, F.; SÁ, J. C. M. Propriedades físicas do solo sob plantio direto influenciadas por calagem preparo inicial e tráfego. Revista Brasileira de
Ciência do Solo, v. 22, p. 301-309, 1998. Disponível em: $<$ http://sbcs.solos.ufv.br/solos/revistas/v22n 2a16.pdf>. Acesso em: 12 mai. 2013. 